\title{
Editorial „Strukturveränderungen in der beruflichen Ausbildung“"
}

\author{
Kai Maaz $\cdot$ Hans-Peter Blossfeld
}

Online publiziert: 28. Mai 2019

(C) The Editors of the Journal 2019

Das deutsche Berufsbildungssystem, das sich in die drei großen Bereiche Übergangssystem, duales System und vollzeitschulisches Ausbildungssystem differenziert, unterliegt permanenten Veränderungen. Es wird von einer Reihe von Rahmungen beeinflusst, auf die es ständig reagieren muss. Das Übergangssystem als System der Berufsvorbereitung zwischen Schule und Berufsausbildung beispielsweise, das in der ersten Hälfte des letzten Jahrzehnts massiv expandierte, ist im Zuge des demographischen Wandels und des wirtschaftlichen Booms der letzten Jahren in Deutschland wieder deutlich geschrumpft. Ferner muss das Berufsbildungssystem auf sich verändernde Anforderungen im Beschäftigungssystem reagieren. Diese nach wie vor enge Verbindung von Ausbildungs- und Beschäftigungssystem trägt gleichzeitig aber auch zu relativ friktionsfreien Übergängen in den Arbeitsmarkt und geringer Jugendarbeitslosigkeit bei. Anpassungsherausforderungen gibt es darüber hinaus aus Veränderungen im Bildungsverhalten im Bereich der allgemeinbildenden Schulen. So ist der Anteil der Schülerinnen und Schüler, die nach der Grundschule auf den nicht-akademischen Zweig des Sekundarbereichs I wechseln und dann nach ihrer Schulausbildung mehrheitlich in das berufliche Bildungssystem einmünden, über die Kohorten kontinuierlich kleiner geworden. Dieser Trend weg von der Berufsausbildung und hin zur akademischen Bildung wird noch dadurch verstärkt, dass ein steigender Anteil der Absolventen des nicht-akademischen Zweigs schulischer Bildung nach der regulären Schullaufbahn eine Hochschulzugangsbe-

Prof. Dr. K. Maaz $(\bowtie)$

DIPF | Leibniz-Institut für Bildungsforschung und Bildungsinformation,

Rostocker Straße 6, 60323 Frankfurt am Main, Deutschland

E-Mail: maaz@dipf.de

Prof. Dr. rer. pol. Dr. h. c. H.-P. Blossfeld

Lehrstuhl für Soziologie I, Universität Bamberg, Feldkirchenstr. 21, 96052 Bamberg, Deutschland

E-Mail: hans-peter.blossfeld@uni-bamberg.de 
rechtigung im Sekundarbereich II erwirbt und dann ein Studium aufnimmt. Infolge dieser Entwicklungen wird, oft vorschnell, von einer weiteren Akademisierung des deutschen Bildungssystems gewarnt. Vergessen wird dabei allerdings, dass gleichzeitig der gegenläufige Trend zu beobachten ist, dass ein immer größerer Anteil der Absolventen des akademischen Zweigs schulischer Bildung, der im Sekundarschulbereich II mit der Hochschulreife endet, eben nicht studiert, sondern eine Berufsausbildung absolviert. Ein solcher Trend einer höheren Allgemeinbildung der beruflich Auszubildenden durch ein höheres schulisches Ausbildungsniveau scheint gut mit den wachsenden Anforderungen der Ausbildungsberufe an die kognitiven und sozialen Kompetenzen der Auszubildenden zu harmonieren. Insbesondere die Digitalisierung als zentrale Entwicklung in allen Bereichen der Wissens- und Informationsgesellschaft verändert die Ausbildungsberufe und die Arbeitswelt drastisch und führt zu der Frage, welche Ausbildungsprofile davon besonders betroffen sind, wo Berufsbilder überarbeitet werden müssen und welche Relevanz „Industrie 4.0“ in den verschiedenen Branchen hat.

Dieses Schwerpunktheft beschäftigt sich mit ausgewählten Aspekten dieser vielfältigen Veränderungsprozesse in der beruflichen Ausbildung. In ihrem Stichwortbeitrag „Herausforderungen und Entwicklungsperspektiven der beruflichen Bildung in Zeiten veränderter Rahmenbedingungen" beschreiben Susan Seeber und Jürgen Seifried zunächst die Stärken des deutschen Systems der Berufsausbildung. Sie gehen dann auf Problemlagen und neue Herausforderungen der Beruflichen Bildung ein, die sich unter anderem in der unzureichenden Integration bestimmter sozialer Gruppen in das Berufsausbildungssystem, den Veränderungen des Verhältnisses von beruflicher und akademischer Ausbildung sowie den Strukturwandel von einer produktionsbezogenen Ökonomie zur Wissens- und Dienstleistungsgesellschaft manifestieren. Weiterführend zeigt der Beitrag von Seeber und Seifried auch Perspektiven für die Weiterentwicklung der Berufsausbildung auf.

Der Beitrag von Laura Menze und Anne Christin Holtmann nimmt den Bereich des Übergangsystems in den Blick und konzentriert sich auf die Gruppe der Jugendlichen ohne Mittleren Schulabschluss, die nach dem Verlassen der Schule oft in Maßnahmen im Übergangsbereich des beruflichen Bildungssystems eintreten. Mit der Analyse von Maßnahmen des Übergangssystems im Ausbildungsbereich greift der Beitrag einen Bereich auf, der in den Bundesländern unterschiedlich strukturiert und durch eine große Dynamik gekennzeichnet ist. Der Beitrag widmet sich im Speziellen der Frage, was Schulabgängerinnen und Schulabgänger ohne Mittleren Schulabschluss aus den Übergangsmaßnahmen mitnehmen können. Mit den Daten des Nationalen Bildungspanels wird gezeigt, was sie aus solchen Maßnahmen für ihren weiteren Bildungsweg mitnehmen können. Im Zentrum stehen die Entwicklungen in drei Bereichen: (1) das Nachholen von Schulabschlüssen, (2) das Sammeln von betrieblichen Erfahrungen und (3) die Entwicklung des Bewerbungsverhaltens für Ausbildungsplätze. Menze und Holtmann zeigen, dass durch das Übergangssystem der Ausbildungszugang in der Tat häufig erleichtert wird, jedoch die Jugendlichen die Teilnahme an Übergangsmaßnahmen entsprechend ihrer Ausgangsbedingungen auf unterschiedliche Weise für sich nutzen. Während diejenigen mit vergleichsweise günstigen Ausgangsbedingungen häufiger Schulabschlüsse nachholen, sammeln 
andere mit schlechteren Ausgangsbedingungen eher betriebliche Erfahrungen und verbessern ihr Bewerbungsverhalten.

Einen weiteren Aspekt zu Strukturveränderungen beruflicher Ausbildung greift Pia N. Blossfeld in ihrem Beitrag ,Soziale Herkunft und langfristige Veränderungen der Übergänge zwischen akademischen und nicht-akademischen Karrieren nach dem ersten Bildungsabschluss " auf. Sie betrachtet in langfristiger Perspektive die zunehmende wechselseitige Durchlässigkeit zwischen den akademischen und beruflich orientierten Bildungswegen und den Zusammenhang dieser Entwicklungen mit sozialer Herkunft. Dabei geht sie insbesondere der Frage nach, wie sich die Attraktivität der Berufsausbildung über die Kohorten verändert hat. Wiederum auf Basis des Nationalen Bildungspanels werden im Beitrag Bildungskarrieren von westdeutschen Erwachsenen aus den Geburtskohorten von 1944 bis 1983 rekonstruiert und verglichen. Die Analysen zeigen, dass sich in den letzten Jahrzehnten die Bildungskarrieren der Jugendlichen und jungen Erwachsenen in Bezug auf die berufliche Ausbildung in Deutschland stark verändert haben. Auf der einen Seite ist der Anteil der Jugendlichen mit einem Haupt- bzw. Realschulabschluss, der eine Berufsausbildung absolviert, deutlich gesunken. Diese Absolventen holen auch zunehmend häufiger eine Hochschulzugangsberechtigung nach. Auf der anderen Seite ist aber auch der Anteil der Studienberechtigten stark gewachsen, der statt eines tertiären Abschlusses eine berufliche Ausbildung absolviert. Der Wechsel vom akademischen zum beruflichen Bildungszweig hat also ebenfalls langfristig stark zugenommen. Die Berufsausbildung scheint deswegen nicht grundsätzlich an Attraktivität verloren zu haben. Vielmehr haben die Auszubildenden im Vergleich zu früher heute oft eine bessere Allgemeinbildung, was den wachsenden Anforderungen in der Berufsausbildung und im Beruf in einer modernen Dienstleistungsgesellschaft durchaus entgegenkommt.

Letztlich wird im Beitrag von Matthias Becker und Georg Spöttl eine Thema bearbeitet, das das gesamte Bildungssystem vor Herausforderungen stellt und das Schule und Ausbildung nachhaltig verändern wird. In ihrem Beitrag ,Auswirkungen der Digitalisierung auf die berufliche Bildung am Beispiel der Metall- und Elektroindustrie" untersuchen sie den spezifischen Aspekt der Digitalisierung für die berufliche Ausbildung. Sie beleuchten die Entwicklung der erneuten Hinwendung zur industriellen Arbeit und beschreiben neue Chancen und Risiken, die unter dem Einfluss der Digitalisierung für die beruflichen Bildung offensichtlich werden. Chancen für Stabilisierung und Weiterentwicklung des Berufsbildungssystems liegen der Anreicherung qualifizierter Arbeit und die Profilierung einiger Berufe. Risiken können durch eine Entwertung bestimmter beruflicher Tätigkeiten infolge von Automatisierungsprozessen entstehen, die einzelne Berufe überflüssig machen können. In diesem Zusammenhanf sind Fragen zu beruflichen Kompetenzen und notwendigen Berufsprofilen für die digitalisierte Arbeit von besonderem Interesse und werden von Becker und Spöttl ausgehend von empirischen Befunden der bayme vbm Studie analysiert und diskutiert. 